\title{
Author Correction: Thermodynamic uncertainty relations constrain non-equilibrium fluctuations
}

Jordan M. Horowitz (D) and Todd R. Gingrich (iD)

Correction to: Nature Physics https://doi.org/10.1038/s41567-019-0702-6, published online 11 November 2019.

In the version of this Perspective originally published, in Box 1, the sentence "The single particle in the high-energy site can move to the lower-energy site with rate $p$, but in doing so the system will lose energy $\Delta E$." has been amended to read "The single particle in the site with high energy $E_{1}$ can move to the site with lower energy $E_{2}$ with rate $p$, but in doing so the system will lose energy $\Delta E=E_{1}-E_{2}$ ". Accordingly, also in Box 1 , the following equation changes have been made. (1) In the equations for ' $\frac{\alpha}{\gamma}$ ' and ' $\frac{\beta}{\delta}$, the superscripts ' $\mu_{\text {left }} / k_{\mathrm{B}} T^{\circ}$

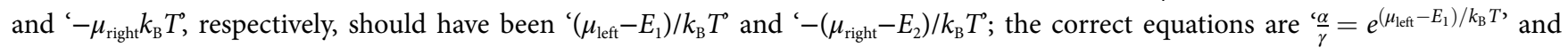
$\frac{\beta}{\delta}=e^{-\left(\mu_{\mathrm{right}}-E_{2}\right) / k_{\mathrm{B}} T}$. (2) In the equation in the sentence beginning "For example:", the term ' $+\frac{\Delta E}{k_{\mathrm{B}} T}$ ' should not have been included; the correct equation is:

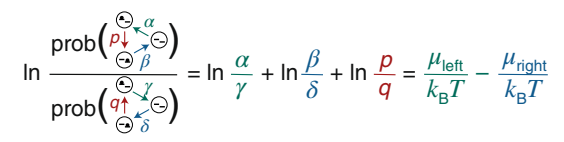

The online versions of the Perspective have been amended.

Published online: 9 March 2020

https://doi.org/10.1038/s41567-020-0853-5

(C) Springer Nature Limited 2020

\section{Author Correction: Half-integer level shift of vortex bound states in an iron-based superconductor}

Lingyuan Kong (D), Shiyu Zhu DiD, Michał Papaj, Hui Chen, Lu Cao (D), Hiroki Isobe, Yuqing Xing, Wenyao Liu, Dongfei Wang (D), Peng Fan, Yujie Sun ID, Shixuan Du, John Schneeloch, Ruidan Zhong, Genda Gu, Liang Fu, Hong-Jun Gao (D) and Hong Ding (D)

Correction to: Nature Physics https://doi.org/10.1038/s41567-019-0630-5, published online 19 August 2019.

In the version of this Article originally published, in the Acknowledgements the sentence "L.F. and G.D.G are supported by US DOE (DE-SC0010526 and DE-SC0012704, respectively)." was incorrect and should have read "The work at MIT is supported by DOE Office of Basic Energy Sciences, Division of Materials Sciences and Engineering under award no. DE-SC0019275. G.D.G. is supported by US DOE DE-SC0012704." This has now been corrected in the online versions of the Article.

Published online: 13 March 2020

https://doi.org/10.1038/s41567-020-0870-4

(c) The Author(s), under exclusive licence to Springer Nature Limited 2020 